\title{
The Coagulation Analysis of Reveals Novel Biomarkers for Neonatal Hyperbilirubinemia in Full Term Newborn
}

\section{Fangye Zhou ( $D$ 345837400@qq.com )}

Chengdu Second People's Hospital

Li Liu

Chengdu Second People's Hospital

Rong Fu

Chengdu Second People's Hospital

\section{Research}

Keywords: coagulation test, neonatal hyperbilirubinemia, Correlation

Posted Date: July 26th, 2021

DOI: https://doi.org/10.21203/rs.3.rs-723930/v1

License: (1) (i) This work is licensed under a Creative Commons Attribution 4.0 International License.

Read Full License 


\section{Abstract}

Background: The coagulation test is used to evaluate in various diseases. Little is known about correlation between the coagulation test and neonatal hyperbilirubinemia. The aim of the study was to determine the neonatal coagulation analysis value in infants with neonatal hyperbilirubinemia as reliable markers.

Methods: All newborns were born and inpatients in the newborn wards of the Chengdu Second People's Hospital. Measurement of serum bilirubin was done to diagnose hyperbilirubinemia. All neonates involved were full term of either gender without any significant illness or major congenital malformations. Data were collected from newborns who had neonatal hyperbilirubinemia and healthy in Chengdu Second People's Hospital. Prothrombin time(PT), Thrombin time(TT), Fibrinogen(Fbg), activated partial thromboplastin time(APTT), and calculated international normalized ration(INR) values were recorded. Linear relationship between INR and level of total bilirubin and indirect bilirubin were analyzed by linear regression. The receiver operating curve(ROC) and the area under the curve(AUC) were made evaluated.

Results: In this case-control study, the mean PT, APTT level were significant higher in the infants with hyperbilirubinemia group compared to the healthy infants. We found the positive correlation between INR and total bilirubin $(R=0.3327 ; P<0.0001)$. Furthermore, there was also significant positive correlation between INR and indirect bilirubin $(R=0.3402 ; P<0.0001)$. Meanwhile, INR in neonates with hyperbilirubinemia significantly achieved an AUC of $0.800(95 \% \mathrm{Cl}=0.6288-0.9712$, cutoff value 1.060 , specificity $71.43 \%$, and sensitivity $80.00 \%$ ).

Conclusions: PT, APTT were significant high in neonatal hyperbilirubinemia group. Correlation were observed between the INR and neonatal hyperbilirubinemia. Importantly, the study revealed INR as novel biomarkers for diagnosis of neonatal hyperbilirubinemia in full term newborn.

\section{Introduction}

Neonatal hyperbilirubinemia was a common problem in newborns[1]. Hyperbilirubinema was a major risk factor for brain damage, bilirubin-induced neurologic dysfunction[2]. It was manifested through yellowish change of skin and mucous membrane[3].

And neonatal jaundice induced blood incompatibility[4]. Some studies have shown the correlation of neonatal hyperbilirubinemia include G6PD deficiency, serum vitamin D level, ASO haemolytic disease[46]. Some causes of significant hyperbilirubinemia were relation with erythrocyte membrane defects, red blood cell enzyme deficiencies[7, 8]. For coagulation system, the interaction of coagulation factors has been found to go beyond their traditional effect and to affect some diseases $[9,10]$. So we appreciated the correlation between hyperbilirubinemia and coagulation system.

The present study was therefore to determine the relationship between PT, PATT, Fbg, TT and hyperbilirubinemia in full term neonates. Through the coagulation analysis, we reveals novel biomarkers 
for the diagnosis of neonatal hyperbilirubinemia in full term newborn.

\section{Methods}

\section{Material and methods}

This study was conducted in Chengdu, Sichuang Province, China, from October 2019 through September 2020. All newborns were born and inpatients in the newborn wards of the Chengdu Second People's Hospital. The birth weight of full term neonates were included in the study. All neonates involved were full term of either gender without any significant illness or major congenital malformations. The neonates with conditions that could aggravate hyperbilirubinemia, such as sepsis, respiratory distress syndrome, asphyxia, diabetic mothers, or intrauterine growth retardation, were excluded from the study. In this casecontrol study, 40 eligible infants with significant hyperbilirubinemia were compared with 30 healthy infants. Additionally, written consent was obtained from all of the parents before any procedures were performed. Hyperbilirubinemia was determined by testing serum bilirubin and clinical manifestation on the second day before they were discharged from the hospital. Increasing of the total bilirubin level more than $34 \mathrm{umol} / \mathrm{L}$ was considered as hyperbilirubinemia. Venous blood samples were collected and sent for prothrombin time(PT), Thrombin time(TT), Fibrinogen(Fbg), activated partial thromboplastin time(APTT), and calculated international normalized ratio(INR).

\section{Statistical analysis}

Statistical analysis Data was entered and analysed using GraphPad Prism 8. Mean and standard deviation were used for the analysis of the data. The unpaired test was used to compare the two groups and line regression to compare the correlation in terms of the Pearson's Correlation Coefficient. For interpretation of results, significance was adopted at $\mathrm{P}<0.05$.

\section{Results}

\section{Clinical characteristics}

In the study, we selected 70 newborns to evaluate in two groups, including 40 neonatal hyperbilirubinemia infants in the case group and 30 healthy neonatal infants in the control group. No asphyxia and inflammation occurred in 70 newborns. Baseline clinical characteristics were similar in the two groups(Table 1). But there was significant difference in WBC, PLT, HGB in the two groups(Table 1). The mean WBC in hyperbilirubinemia group $\left(12.98 \pm 4.67 \times 10^{9} / \mathrm{L}\right)$ was significantly higher than that of the control group $\left(6.55 \pm 1.63 \times 10^{9} / \mathrm{L}\right)(\mathrm{P}<0.0001)$. The mean PLT in hyperbilirubinemia group $(329.25 \pm$ $\left.76.77 \times 10^{9} / \mathrm{L}\right)$ was significantly higher than that of the control group $\left(255.13 \pm 38.11 \times 10^{9} / \mathrm{L}\right)(\mathrm{P}<0.0001)$. The mean HGB in hyperbilirubinemia group $(162.15 \pm 17.41 \mathrm{~g} / \mathrm{L})$ was significantly higher than that of the control group $(134.33 \pm 11.25 \mathrm{~g} / \mathrm{L})(\mathrm{P}<0.0001)$. 
Table 1. Clinical characteristics between the significant neonatal hyperbilirubinemia groups and the control groups

\begin{tabular}{|c|c|c|c|}
\hline \multirow{2}{*}{$\begin{array}{l}\text { Characteristics } \\
\text { P }\end{array}$} & hyperbilirubinemia groups $(\mathrm{n}=40)$ & \multicolumn{2}{|c|}{ control groups $(\mathrm{n}=30)$} \\
\hline & Mean \pm SD & Mean \pm SD & \\
\hline Sex (males/females) & $22 / 19$ & $15 / 15$ & \\
\hline Birth weight $(g)$ & $2487 \pm 980$ & $2536 \pm 783$ & \\
\hline Age (days) & 4. 7 & 5.0 & \\
\hline \multicolumn{4}{|l|}{ Mode of delivery } \\
\hline Vaginal delivery & 22 & 15 & \\
\hline Caesarean delivery & 18 & 15 & \\
\hline $\begin{array}{l}\operatorname{WBC}\left(3.5-9.2 \times 10^{9} / \mathrm{L}\right) \\
<0.0001\end{array}$ & $12.98 \pm 4.67$ & & $6.55 \pm 1.63$ \\
\hline $\operatorname{RBC}\left(4.09-5.74 \times 10^{12} / \mathrm{L}\right)$ & 4. $68 \pm 0.58$ & $4.66 \pm 0.36$ & 0.8819 \\
\hline $\operatorname{PLT}\left(85-303 \times 10^{9} / \mathrm{L}\right)$ & $329.25 \pm 76.77$ & $255.13 \pm 38.11$ & $<0.0001$ \\
\hline $\operatorname{HGB}(131-172 \mathrm{~g} / \mathrm{L})$ & $162.15 \pm 17.41$ & $134.33 \pm 11.25$ & $<0.0001$ \\
\hline
\end{tabular}

\section{Comparison of coagulation test}

When Comparing the neonatal hyperbilirubinemia groups and the control groups using coagulation test, there was significant difference in PT, INR, APTT(Table 2). The mean PT in hyperbilirubinemia group(12.54 \pm 1.39 second) was significantly higher than that of the control group(11.3 \pm 0.63 second)(P $<0.0001)$. The mean INR in hyperbilirubinemia group $(1.14 \pm 0.13)$ was significantly higher than that of the control group $(1.03 \pm 0.06)(P=0.0001)$. The mean APTT in hyperbilirubinemia group $(47.1 \pm 8.89$ second) was significantly higher than that of the control group $(27.67 \pm 1.97$ second $)(P<0.0001)$.

Table 2. Comparison of various characteristics between the significant neonatal hyperbilirubinemia groups and control groups 


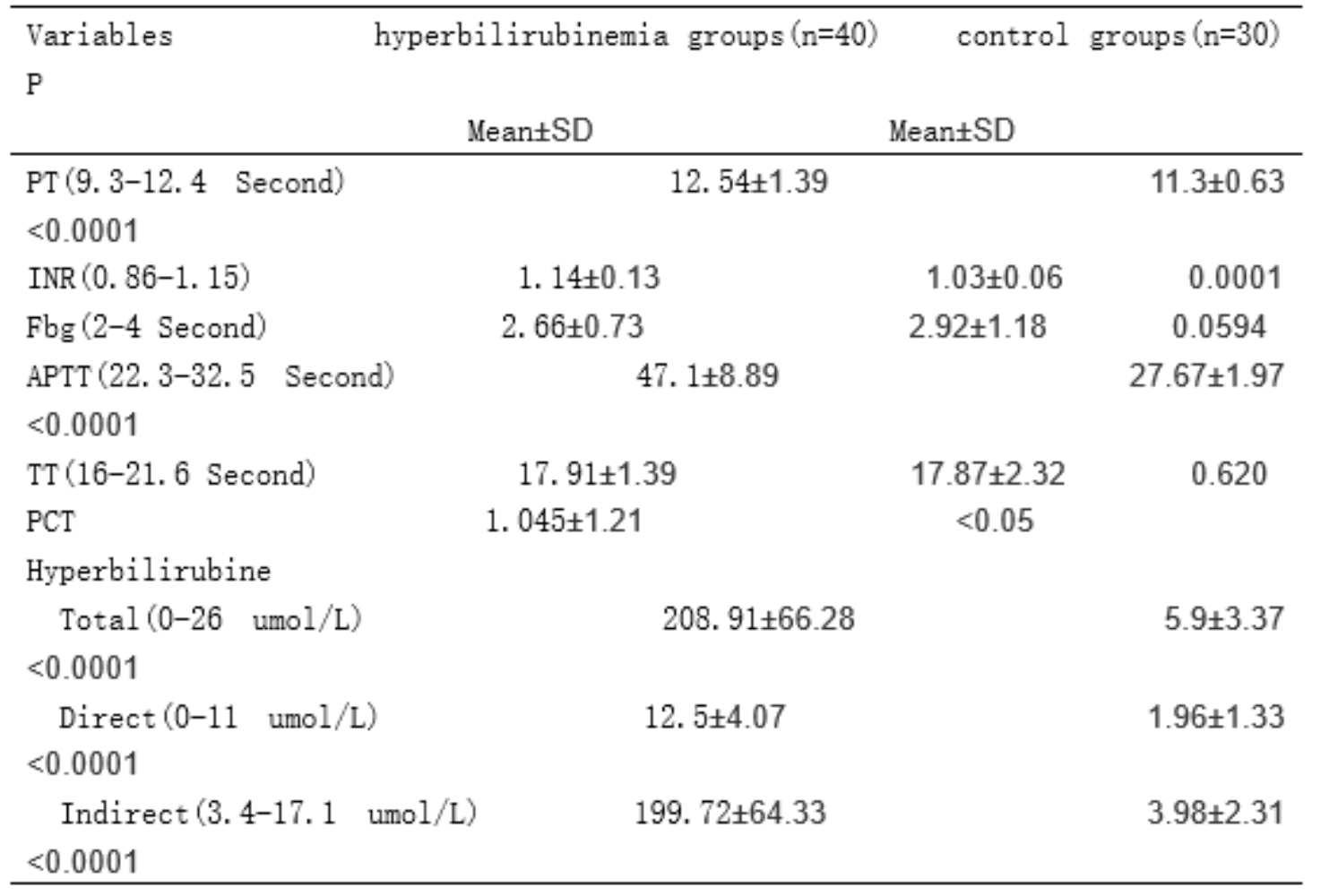

\section{the relationship was observed between INR and level of total bilirubine}

As shown, the relationship was observed between INR and level of total bilirubine $(R=0.3327 ; P<0.0001)$ (Fig. 1). As shown in Fig. 2, the relationship was also observed INR and level of indirect bilirubin( $R=$ 0.3406; $\mathrm{P}<0.0001$ )(Fig. 2). The efficacy of detection of INR was reported using the ROC AUC phenomenon in neonates with hyperbilirubinemia. INR in neonates with hyperbilirubinemia significantly achieved an AUC of $0.800(95 \% \mathrm{Cl}=0.6288-0.9712$, cutoff value 1.060 , specificity $71.43 \%$, and sensitivity 80.00\%)(Fig. 3).

\section{Discussion}

The study found the relationship between coagulation test and hyperbilirubinemia in full term infants. The mean PT, APTT level were significant higher in the infants with hyperbilirubinemia group compared to the healthy infants. At the same time, the mean serum PCT level was higher in the infants with hyperbilirubinemia group. In our study, the WBC, PLT and HGB level in hyperbilirubinemia group were higher than the healthy group $(P<0.0001)$. We found there is the correlation between INR and total bilirubin $(R=0.3327 ; P<0.0001)$. There is also correlation between INR and indirect bilirubin $(R=0.3406 ; P$ $<0.0001)$.

Hyperbilirubinemia often occurred in infants without apparent reason[11]. The causes of hyperbilirubinemia and effective prevention strategies have recommended as clinical guidelines[12]. The American Academic of Pediatrics(APP) recommends a follow-up visit after $48-72 \mathrm{~h}$ of the newborns discharged[13]. For the main cause of neonatal hyperbilirubinemia, a large of study have received developments[14]. Compared with other study, we used INR to evaluate the correlation between 
hyperbilirubinemia and coagulation. The coagulation system have easily activated by inflammation, severe disease in human[15]. Therefore, in this study, we evaluated the hyperbilirubinemia and health groups, PT and APTT were significantly prolonged in infants with hyperbilirubinemia than health control and there was significant difference $(P<0.0001)$.

The efficacy of detection of INR was reported using the ROC AUC phenomenon in neonates with hyperbilirubinemia. INR in neonates with hyperbilirubinemia significantly achieved an AUC of $0.800(95 \%$ $\mathrm{Cl}=0.6288-0.9712$, cutoff value 1.060 , specificity $71.43 \%$, and sensitivity $80.00 \%$ ).

\section{Conclusion}

In Conclusion, our study suggested that hyperbilirubinemia in infants may induce coagulation system to change. Maybe, the change of coagulation system may be a cause of early significant hyperbilirubinemia. Moreover, it appears to have a pathogenic potential in newborns, high PLT may be a potential problem. Importantly, we may consider coagulation system when hyperbilirubinemia was observed. We recommend the INR as novel biomarkers for diagnosis of neonatal hyperbilirubinemia in full term newborn.

\section{Abbreviations}

PT: Prothrombin time, TT: Thrombin time, Fbg: Fibrinogen, APTT: activated partial thromboplastin time, INR: international normalized ration, WBC: White blood cell, RBC: Red blood cell, PLT: Platelet, HGB: Hemoglobin, APP: The American Academic of Pediatrics

\section{Declarations}

\section{Ethics approval and consent to participate}

The study was approved by the Ethics Committee of Chengdu second people's hospital, China and conducted according to the principles in the Declaration of Helsinki. Written informed consent was acquired from the patient and his parents to allow his datasets being used for scientific purposes.

\section{Availability of data and materials}

Not applicable

\section{Authors' contributions}

Zhou Fangye: Conceived and designed the experiments, analyzed data and drafted the manuscript; Liu Li: Collected the data and helped in data analysis; Fu Rong: collected the data.

\section{Funding}


Not applicable

\section{Competing interests}

The authors have no conflicts of interest to declare.

\section{Acknowledgements}

Not applicable

\section{References}

1. Gutta, S; Shenoy, J; Kamath, SP; Srinivasan, M; Mithra, P; Baliga, BS; Sarpangala, M. Light Emitting Diode (LED) Phototherapy versus Conventional Phototherapy in Neonatal Hyperbilirubinemia: A Single Blinded Randomized Control Trial from Coastal India. Biomed Res Int. 2019V2019N:6274719

2. Johnson L, Bhutani VK. The clinical syndrome of bilirubin induced neurologic dysfunction. Semin Perinatol. 2011;35: 101-13.

3. Delaney M, Matthews DC. Hemolytic disease of the fetus and newborn: managing the mother, fetus, and newborn. Hematol Am Soc Hematol Educ Program. 2015;1:146-51.

4. Das, S; Shastry, S; Chakravarthy, PK; Baliga, PB. Clinical Implication of Immunohaematological Tests in ABO haemolytic disease of newborn: Revisiting an old disease. Transfus Med. 2020:1-6.

5. Mehrpisheh, S; Memarian, A; Mahyar, A; Valiahdi, NS. Correlation between serum vitamin D level and neonatal indirect hyperbilirubinemia. BMC Pediatr. 2018V18N1:178.

6. Prabhakar, N; Ahuja, CK; Khandelwal, N. B/L Basal Ganglia Lesions in a Child Leading to a Diagnosis of Glucose-6-Phosphate Dehydrogenase Deficiency. Ann Neurosci. 2018V25N1:50-52.

7. Brito, MA; Sliva, RM; Matos, DC; Da Silva, AT; Brites, DT. Alterations of erythrocyte morphology and lipid composition by hyperbilirubinemia. Clin Chim Acta. 1996V249N1-2:149-65.

8. Rets A; Clayton, AL; Christensen, RD; Agarwal, AM. Molecular diagnostic update in hereditary hemolytic anemia and neonatal hyperbilirubinemia. Int J Lab Hematol. 2019V41 Suppl 1N:95-101.

9. Göbel, K; Eichler, S; Wiendl, H; Chavakis, T; Kleinschnitz, C; Meuth, SG. The Coagulation Factors Fibrinogen, Thrombin, and Factor XII in Inflammatory Disorders-A Systematic Review. Front Immunol. 2018V9N:1731.

10. Undas, A; Brummel-Ziedins, KE; Mann, KG. Anticoagulant effects of statins and their clinical implications. Thromb Haemost. 2014V111N3:392-400

11. Chou, JH. Predictive Models for Neonatal Follow-Up Serum Bilirubin: Model Development and Validation. JMIR Med Inform. 2020V8N10:e21222.

12. Mandour, YM; El Sayed, MA; El Sayed Morgan, A; Bassam, R; Fadl, H; Elrefae, A. Audiological assessment of neonatal hyperbilirubinemia. Int J Pediatr Otorhinolaryngol. 2020V135N:110126 
13. Khairy, MA; Abuelhamd, WA; Elhawary, IM; Mahmoud Nabayel, AS. Early predictors of neonatal hyperbilirubinemia in full term newborn. Pediatr Neonatol. 2019V60N3:285-290.

14. Alkén, J; Håkansson, S; Ekéus, C; Gustafson, P; Norman, M. Rates of Extreme Neonatal Hyperbilirubinemia and Kernicterus in Children and Adherence to National Guidelines for Screening, Diagnosis, and Treatment in Sweden. JAMA Netw Open. 2019 Mar; 2(3): e190858.

15. Subramaniam, S; Scharrer, I. Procoagulant activity during viral infections. Front Biosci (Landmark Ed). 2018V23N:1060-1081.

\section{Figures}

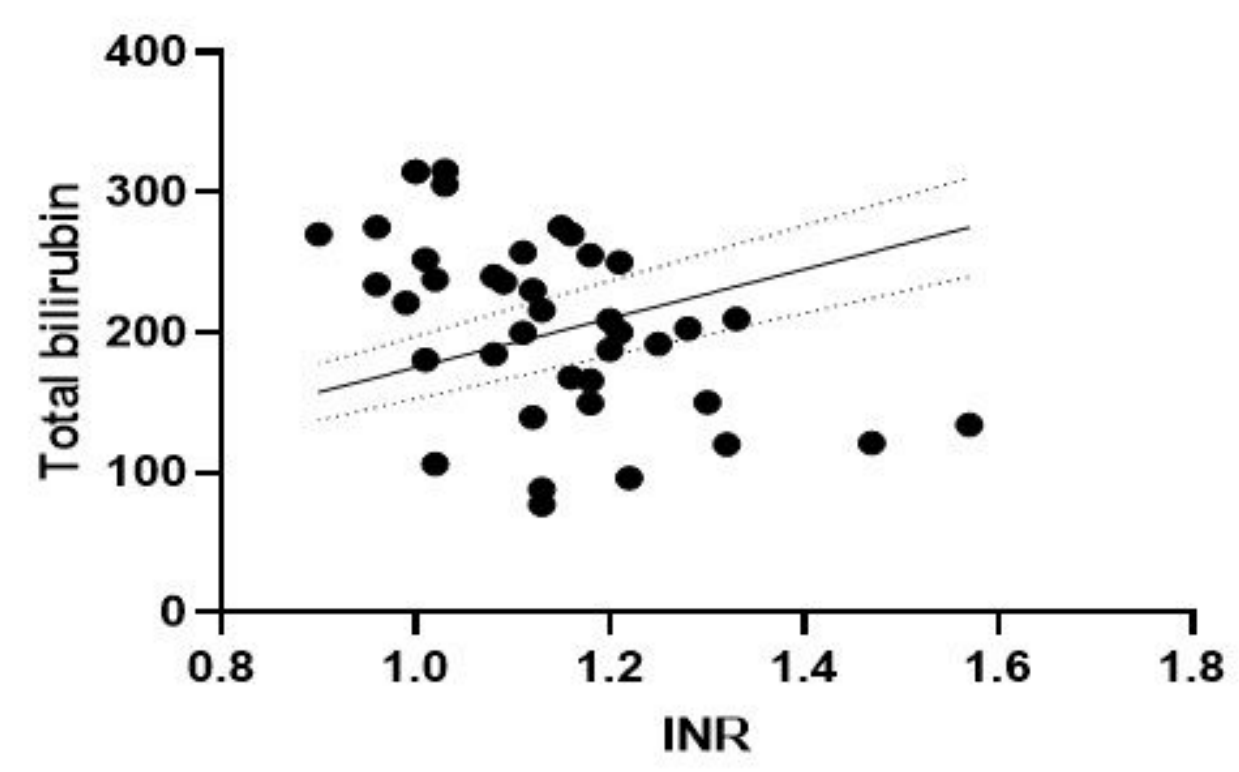

Figure 1

Linear relationship between INR and level of total bilirubin $(R=0.3327 ; P<0.0001)$ 


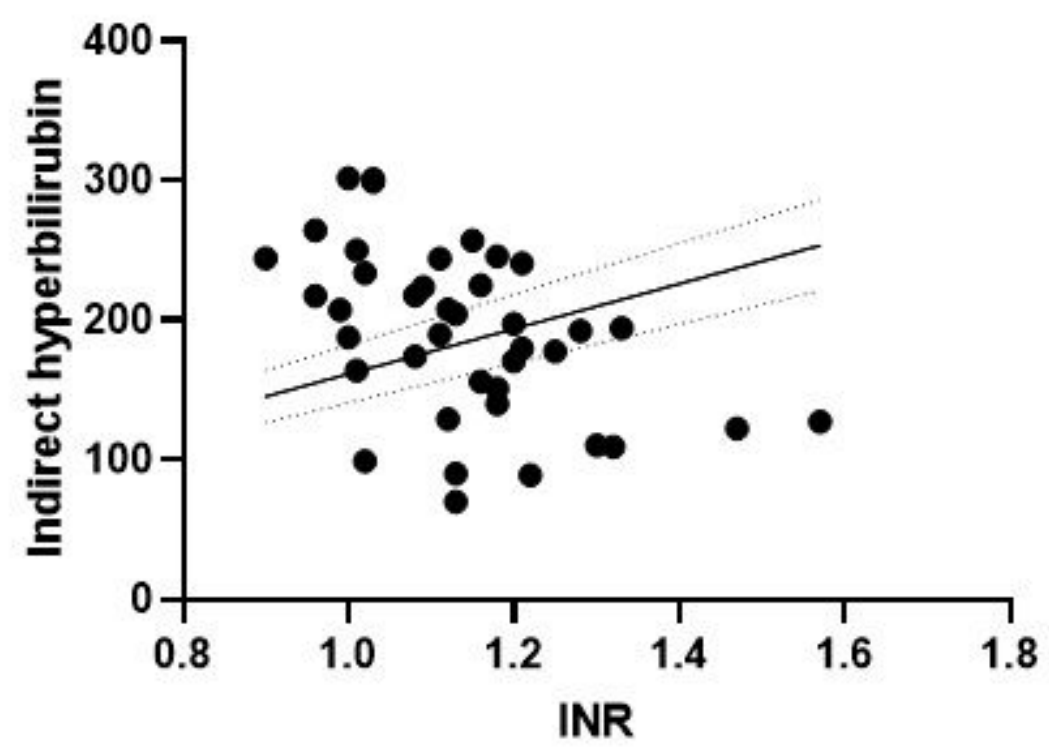

Figure 2

Linear relationship between INR and level of indirect bilirubin $(R=0.3406 ; P<0.0001)$

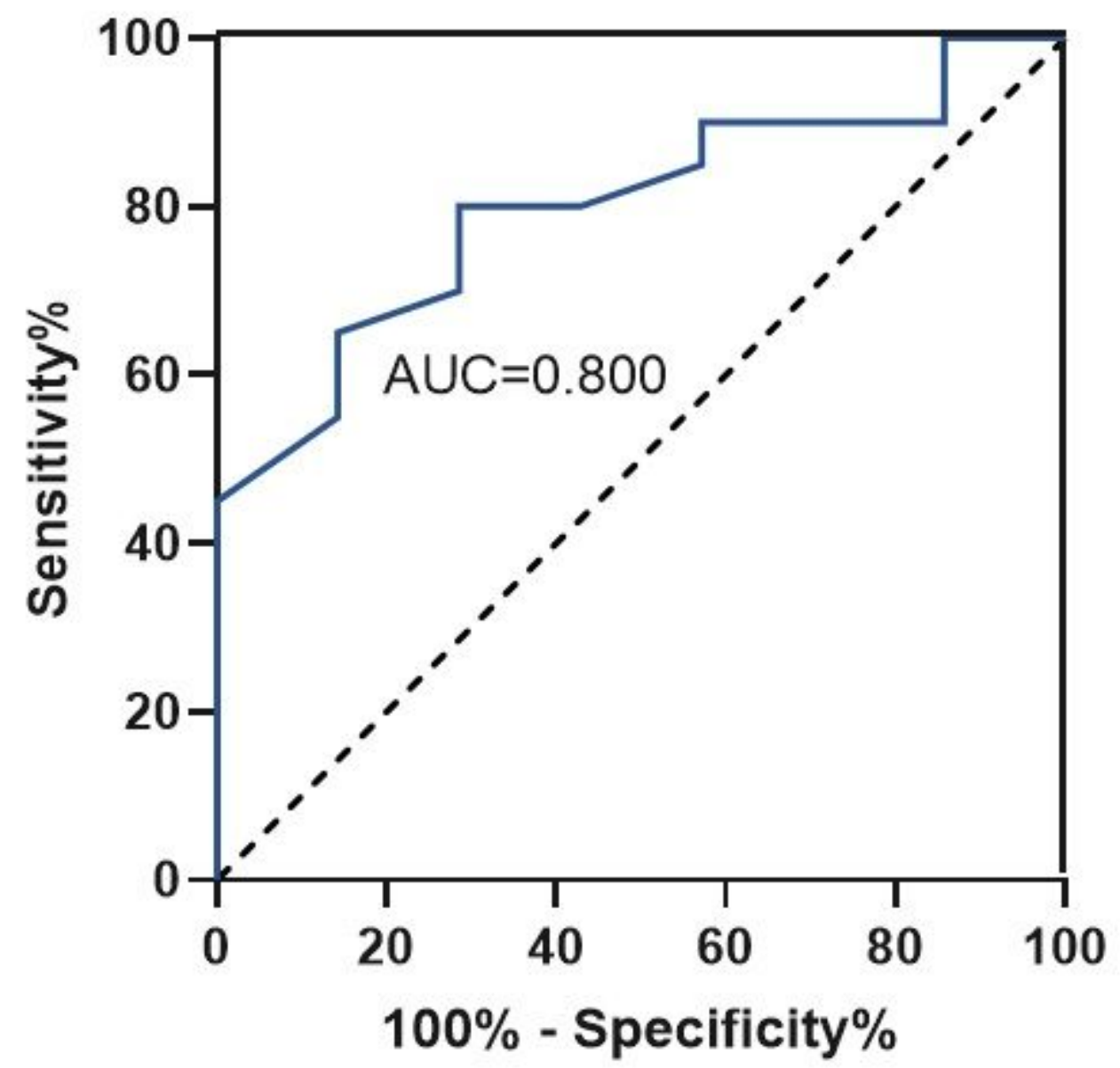


Figure 3

ROC curves of INR for the neonates with hyperbilirubinemia 